\title{
Adamantios Koraïs : la dimension éthique et politique de l'éducation « classique »
}

Adamantios Korais, and the Political and Ethical Dimension the "Classical

Education"



$\varepsilon \kappa \pi \alpha i ́ \delta \varepsilon v \sigma \eta \varsigma »$

\section{Aikaterini Lefka}

\section{(2) OpenEdition}

\section{Journals}

Édition électronique

URL : https://journals.openedition.org/ceb/5726

DOI : 10.4000/ceb.5726

ISSN : 2261-4184

\section{Éditeur}

INALCO

Édition imprimée

Date de publication : 1 mars 2015

ISBN : 978-2-85831-224-5

ISSN : 0290-7402

\section{Référence électronique}

Aikaterini Lefka, « Adamantios Koraïs : la dimension éthique et politique de l'éducation « classique » » Cahiers balkaniques [En ligne], Hors-série | 2015, mis en ligne le 17 novembre 2015, consulté le 06

juillet 2021. URL : http://journals.openedition.org/ceb/5726 ; DOI : https://doi.org/10.4000/ceb.5726

Ce document a été généré automatiquement le 6 juillet 2021.

\section{(c) (†) \&}

Cahiers balkaniques est mis à disposition selon les termes de la Licence Creative Commons Attribution - Pas d'Utilisation Commerciale 4.0 International. 


\title{
Adamantios Koraiis : la dimension éthique et politique de l'éducation « classique »
}

\author{
Adamantios Koraiis, and the Political and Ethical Dimension the "Classical \\ Education"

 \\ $\varepsilon \kappa \pi \alpha i ́ \delta \varepsilon v \sigma \eta \varsigma »$
}

Aikaterini Lefka

\section{Introduction}

1 La $3^{\mathrm{e}}$ Assemblée nationale de Trézène, réunie en 1827 , vote un texte exprimant officiellement à Adamantios Koraiis la reconnaissance de ses compatriotes pour les bienfaits de son activité intellectuelle et morale, étendus sur toute la nation hellénique. Permettez-moi de citer quelques phrases de ce texte :

\begin{abstract}
La Grèce te félicite, co-citoyen excellent, qui te réjouit de sa résurrection. Elle t'est reconnaissante, car elle a bénéficié d'un bienfait inestimable grâce à tes écrits excellents. L'amour des Hellènes pour l'éducation provient des lumières que tes Prolégomènes ont semées dans leur cœur depuis quelques années, des livres que ton patriotisme a procurés à notre pays, des écoles que tes sages conseils ont fondées. De causes aussi bienfaitrices ont apporté les biens de la liberté dont tes co-citoyens profitent aujourd'hui. Des biens aussi inestimables sont dignes de la gratitude des générations et des siècles [...] $]^{1}$.
\end{abstract}

Depuis lors, de nombreux représentants des lettres néohelléniques, ainsi que des instances officielles, comme les universités ou l'État grec ont exprimé leur admiration

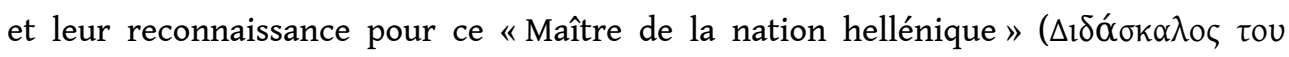
ГÉvous), entièrement dévoué à son œuvre éducative.

Dans le présent article, je tenterai, à la suite de l'analyse de passages pertinents de quelques-uns des textes de Koraïs, de mieux clarifier ses positions sur la manière dont 
l'étude des auteurs "classiques " pouvait contribuer à l'éducation éthique et politique des Grecs, pendant la guerre d'indépendance et après la création de l'État néohellénique. Il me semble que les idées de Koraïs peuvent présenter encore de l'intérêt aujourd'hui, non seulement pour les Grecs, qui traversent actuellement une sérieuse crise économique, politique et éthique, mais aussi pour tout être humain désirant une vie heureuse.

\section{Quelques données biographiques}

4 Adamantios Koraïs (1748-1833), médecin et philologue classique, est un érudit cosmopolite de l'âge des Lumières qui a choisi pendant la plus grande partie de sa vie de vivre à Paris, où il a produit une œuvre philologique et littéraire extraordinaire.

Quand, en 1805, les frères Zossimas, des riches commerçants épirotes, lui posent la question: "Par quel moyen pourrait-on accélérer la renaissance de la Grèce, qui débute?", Koraïs répond: "Par la réunion et l'édition des textes des poètes et des écrivains grecs les plus importants, avec des longues préfaces dans le langage courant".

6 Convaincus, les frères Zossimas décident de financer l'édition de la «Bibliothèque hellénique » dans le cadre de laquelle Koraïs traduit, commente et publie de nombreux textes antiques qu'il estime précieux pour l'éducation morale et politique de la nation grecque, par exemple, des rhapsodies de l'Iliade, des discours d'Isocrate, certaines des Vies parallèles de Plutarque, des œuvres de Platon, de Xénophon, d'Aristote, d'anciens médecins... À ce travail monumental, Koraïs ajoute de nombreuses lettres et des essais, souvent dans le cadre des Préfaces (Prolegomena) de ces œuvres, ou publiés indépendamment, analysant la situation de la Grèce de son temps et $\mathrm{y}$ apportant ses propres conseils.

7 Selon lui, la révolution de 1821 s'est produite quelques décennies trop tôt, car les Grecs avaient pris conscience de leur soif de liberté, de justice et de dignité, mais n'avaient pas eu le temps de s'éduquer suffisamment pour savoir comment réaliser l'organisation efficace d'un État démocratique ${ }^{2}$. Cette attitude lui a valu de nombreuses critiques; cependant, jusqu'à sa mort le savant n'a jamais cessé de soutenir la Révolution et la création du nouvel État grec, matériellement et moralement, directement et indirectement, en mobilisant compatriotes et étrangers.

\section{L'importance de l'éducation}

8 Koraïs distingue les hommes en esprits « ronds » et " carrés ». Les premiers manquent d'éducation et peuvent dès lors "rouler» dans tous les sens, puisque facilement influençables par les avis des uns et des autres. Les seconds, « taillés " par l'éducation, se tiennent solidement ancrés sur leurs principes, car ils ont pleinement conscience de ce qui est vrai et juste ou non, ayant développé leur esprit rationnel et critique ${ }^{3}$.

9 Ce penseur considère comme un "axiome de l'éthique et de la politique » l'idée que «quand les Lumières (c.-à-d. du savoir) brillent, l'esclavage doit nécessairement cesser ", car "les sciences et la tyrannie n'ont jamais cohabité. Si la tyrannie n'a pas réussi à les chasser, elle doit obligatoirement être chassée par elles $»^{4}$. 
10 C'est pourquoi il soutient inconditionnellement et pendant toute sa vie que le meilleur moyen pour changer le statut de la Grèce soumise aux Ottomans en créant sa "renaissance ", c'est l'éducation ${ }^{5}$. Les défenseurs de l'éducation de la nation sont même considérés par le savant comme des personnes « qui coopèrent avec la volonté divine ", laquelle ne peut que soutenir la libération et le redressement des Grecs ${ }^{6}$.

11 Koraïs distingue l'éducation "de la tête » et celle "du cœur». La première sans la deuxième est précieuse, mais elle se résume en une accumulation de connaissances scientifiques, et donc ne garantit pas le bonheur de l'individu, encore moins de toute la nation, le seul bouclier contre l'esclavage. La véritable paideia, considérée comme «le trésor de l'âme", concerne prioritairement l'éducation du caractère ${ }^{7}$ et doit commencer dès la petite enfance, afin que les jeunes gens apprennent à aimer et à suivre la vérité, la liberté et la justice pour toute leur vie ${ }^{8}$.

L'éducation des enfants enseigne et le juste usage de la richesse, et l'honnête soin de la pauvreté, ou la patience philosophique. Elle seule garde le riche de la bêtise de considérer ceux qui sont moins fortunés que lui comme ses inférieurs, autant que le pauvre de flatter les riches et d'atteindre par la ruse et le mensonge ce qu'il doit gagner avec ses peines. Elle seule amène et le moins savant à honorer la sagesse et le sage à ne pas la déshonorer par quelque barbarie bestiale de ses mœurs. Sans elle, l'or dans les mains du riche et les sciences dans la tête du sage deviennent des instruments de destruction plus terribles qu'un couteau dans les mains d'un ivrogne ou d'un fou. Vos enfants, amis grecs, peuvent grâce à leur intellect devenir des astronomes, des chimistes, des physiciens et acquérir toute science et toute connaissance; mais ils vont cueillir davantage de dommages que de bénéfices de celles-ci, si leur éducation depuis l'enfance n'arrive pas à les rendre des êtres humains utiles aux autres'.

12 Seule cette éducation peut encourager la lutte libératrice contre toute forme de tyrannie, y compris la tyrannie de ses propres passions, qui est la plus dangereuse de toutes - résultat inévitable intériorisé d'une vie d'esclavage ${ }^{10}$. De plus, elle peut soutenir la réalisation d'un État bien administré dans la suite, car le peuple apprend à se soumettre seulement aux lois, à respecter ses gouverneurs, ainsi qu'à honorer et à aimer ses concitoyens comme des frères, ses égaux ${ }^{11}$.

Inspiré par la Renaissance italienne, Koraïs soutient que l'étude des grandes œuvres classiques de la littérature grecque et latine est la plus appropriée pour réaliser cette tâche d'éducation éthique et politique, difficile parmi toutes, mais indispensable pour le bonheur privé et public ${ }^{12}$.

\section{Comment l'éducation « classique " peut-elle former le caractère des citoyens?}

Comment l'éducation «classique» peut-elle jouer un tel rôle éthique et politique d'importance capitale?

D'abord, Koraïs pense que «la langue hellénique (...), à côté de ses autres qualités, possède quelque charme exceptionnel, par lequel non seulement elle adoucit les mœurs des jeunes, mais aussi elle les rend plus modestes et plus tempérés ${ }^{13}$. Qui plus est, il croit que l'étude sérieuse du grec ancien et du latin, qu'il considère indissociable du grec $^{14}$, aidera les Hellènes de son temps à avoir une meilleure connaissance de leur propre langue maternelle à usage courant, qui a besoin de nouvelles œuvres littéraires 
pour mieux se définir et se développer. Il s'agit d'une tache d'importance primordiale, car la langue nationale est l'expression de la pensée et de la conscience d'un peuple ${ }^{15}$.

En deuxième lieu, l'étude des textes classiques enseigne « aux ignorants la gloire de nos ancêtres, et les erreurs, par lesquels une telle gloire a péri, ainsi que les moyens, par lesquels on peut à nouveau l'assumer, moyens qui sont fondés sur la véritable Éthique $»^{16}$.

17 Enfin, ce n'est pas seulement le contenu théorique des œuvres de l'extraordinaire culture de l'Antiquité qui peut offrir aux jeunes des bases éthiques et politiques solides, mais aussi et surtout l'exemple concret des personnages historiques qui incarnent ces valeurs. Les Grecs et les Romains illustres du passé doivent devenir des modèles de vertu, de justice, d'égalité et d'amour envers la patrie, des exemples à suivre, comme, par ailleurs, ceux des personnalités extraordinaires contemporaines, rencontrées chez d'autres peuples ${ }^{17}$.

Une telle vertu demandait beaucoup de ses disciples le vertueux Épictète et Socrate qu'Épictète admirait; elle sera acquise par celui qui imitera ces hommes bienheureux de manière sage, ayant comme seul but de cette acquisition de faire du bien à son pays, en bénéficiant lui-même le premier, et de la rendre bienheureuse par son propre bonheur ${ }^{18}$.

Pour atteindre ces objectifs, il faut évidemment que l'enseignant des textes « classiques » suive une méthodologie appropriée et bien réfléchie. Koraïs s'est penché avec beaucoup d'attention sur cette question concrète et développe à plusieurs reprises des conseils à suivre.

19 Il pense d'abord que la philologie doit aller de pair avec la philosophie : la sagesse n'est pas une accumulation de connaissances, mais un véritable " art de vivre », menant à la vertu et au bonheur, d'après les maîtres de l'Antiquité19. L'enseignant doit effectuer un choix approprié de textes à étudier avec les jeunes; ceux-ci doivent s'accorder aux principes éthiques et politiques recherchés ${ }^{20}$.

Koraïs insiste sur la nécessité d'intégrer des méthodes d'enseignement modernes, comme celles de Pestalozzi ${ }^{21}$, d'utiliser une langue compréhensible et de se concentrer sur l'essentiel de la matière à transmettre, en évitant autant le perfectionnisme stérile que le manque de connaissances élémentaires ${ }^{22}$. Enfin, le savant n'omet pas de mentionner comme des conditions sine qua non de l'enseignement réussi l'abandon des punitions physiques qui ne font que perpétuer les mœurs des esclaves, et l'effort pour enseigner de manière agréable, en encourageant une émulation saine et spontanée ${ }^{23}$. Il va jusqu'à soutenir que l'enseignant doit aimer et traiter ses étudiants comme ses propres enfants ${ }^{24}$.

21 Ainsi les jeunes arriveront-ils « à lire les recommandations des hommes anciens, non pas comme des lecteurs, mais comme des auditeurs de maîtres présents. Cette manière seule peut les inciter à les imiter, seule peut engendrer dans leurs âmes l'amour de la vraie liberté $»^{25}$.

\section{Conclusions}

22 Les chercheurs contemporains semblent divisés en ce qui concerne l'importance de la dimension éthique et politique accordée par Koraïs aux textes qu'il a traduits et édités ${ }^{26}$. Il me semble que, si nous nous penchons avec attention sur les écrits de ce savant, comme nous l'avons fait dans le cadre de la présente étude, nous pouvons 
attester du rôle primordial de cette double finalité concernant l'étude de textes « classiques » de l'Antiquité. Je pense, de surcroît, que les idées de Koraïs peuvent être largement appliquées encore maintenant, dans un monde qui cherche les moyens de sortir d'une crise non seulement économique, mais surtout éthique, politique, sociale, voire métaphysique.

Je clôturerai avec une citation d'Angelos Terzakis, qui exprime, à mon avis, d'une manière particulièrement poétique, la quintessence de l'œuvre inestimable d'Adamantios Koraïs :

Koraïs : une âme d'ermite, derrière la fenêtre d'un pays étranger, dans la nuit. Il veille et il illumine ${ }^{27}$.

\section{BIBLIOGRAPHIE}

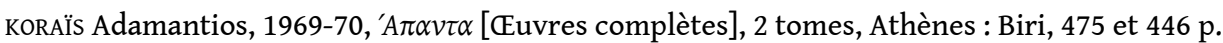

\section{NOTES}

1. $\Psi \eta ́ \varphi ı$

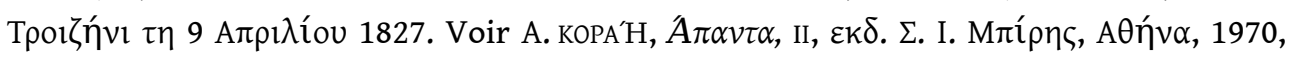
p. 8 (la traduction de tous les textes est de l'auteur).

2. Voir, par exemple, la « lettre confidentielle " que Koraïs adressa aux dirigeants des Grecs le 2 août 1825 (A. KOPA'H, op. cit., II, p. 255-261).

3. Voir la lettre de Koraïs à A. Kontostavlos, du 25 octobre 1822 (A. KopA'H, op. cit., II, p. 203).

4. Voir la lettre de Koraïs à A. Idromenos de Parga, du 27 novembre 1803 (А. KOPA'H, ibid., I, p. 354).

5. Voir « L'art de la parole », Bibliothèque hellénique, Préface $\mathrm{n}^{\circ}$ 1, 1807 (А. KорA'H, ibid., I, p. 275-276).

6. Voir « Opinions pédagogiques », Lettres, III, 605 (A. KopAń, ibid., I, p. 392).

7. Voir « Opinions pédagogiques », Lettres, III, 397 (A. KOPA'H, ibid., I, p. 392).

8. Voir « Opinions pédagogiques », Lettres, III, 547 (A. KOPA'H, ibid., I, p. 392).

9. Voir « L'éducation des enfants », Pensées improvisées (A. KoPA'H, ibid., I, p. 295).

10. Voir la lettre de Koraïs aux gouverneurs de la Grèce, du 10 janvier 1822 (А. коРA'H, ibid. II, p. 174-175).

11. Voir « Opinions pédagogiques », Lettres, III, 932 (А. KOPA'H, ibid., I, p. 392-393).

12. Voir, par exemple, "L'art de la parole ", Bibliothèque hellénique, $\mathrm{n}^{\circ} 1$, Préface, 1807 (A. KOPA'H, ibid., I, p. 268-269) et «L'éducation des enfants ", Pensées improvisées (A. KOPA'H, ibid., I, p. 297). 
13. Lettre de Koraïs à A. Vassileiou, de 1804, où il expose les rapports entre le grec ancien et moderne (A. KOPA'H, ibid., I, p. 339).

14. Voir, par exemple, «l'Art de la parole », Bibliothèque hellénique, $\mathrm{n}^{\circ}$ 1, Préface, 1807 (А. КОРА'H, ibid., I, p. 274 et 276).

15. Voir A. KORAïs, « Aelianus ", Avant-garde de la bibliothèque hellénique, 1805 (А. KOPA'H, ibid., I, p. 194).

16. Voir les remarques de Koraïs notamment concernant l'enseignement d'œuvres de rhétorique, mais valables également pour toute la littérature de l'antiquité, dans " l'Art de la parole ", in Bibliothèque hellénique, Préface $n^{\circ}$ 1, 1807 (A. KOPA'H, ibid., I, p. 262).

17. Voir, par exemple, la lettre de Koraïs aux représentants des Grecs envoyés en mission à Londres, du 21 février 1824 (A. KOPA'H, ibid., II, p. 238-239) et idem, "Socrate ", Bibliothèque hellénique, ${ }^{\circ}$ 15, Préface, 1825 (А. коРA'H, ibid., I, p. 35-37).

18. A. KORAïs, «Épictète », Bibliothèque hellénique, $\mathrm{n}^{\circ}$ 7, Préface, 1827 (А. KOPA'H, ibid., I, p. 160).

19. Voir, par exemple, la lettre de Koraïs à E. Kaïri de Kydonia, du 18 janvier 1815 (A. KopA'H, ibid., II, p. 294-297), « Aux Hellènes ", Avant-garde de la bibliothèque hellénique, 1805 (А. KоРА'H, ibid., I, p. 248-249, 254) et «Chercheurs et législateurs de la langue ", Bibliothèque hellénique, $\mathrm{n}^{\circ}$ 6, Préface, 1812 (А. KOPA'H, ibid., I, p. 310-312).

20. Voir, par exemple, "Aux Hellènes ", Avant-garde de la bibliothèque hellénique, 1805 (A. KOPA'H, ibid., I, p. 243-244, 248, 252, 259).

21. Voir "La Communauté de Constantinople", Pensées improvisées, Bibliothèque hellénique, $\mathrm{n}^{\circ}$ 4, Préface, 1810 (А. KOPA'H, ibid., I, p. 280-281).

22. Voir, par exemple, «Aux Hellènes », Avant-garde de la bibliothèque hellénique, 1805 (A. KOPA'H, ibid., I, p. 257-258).

23. Voir, par exemple, "Aux Hellènes ", Avant-garde de la bibliothèque hellénique, 1805 (A. KOPA'H, ibid., I, p. 250-251).

24. Voir, par exemple, "Aux Hellènes ", Avant-garde de la bibliothèque hellénique, 1805 (A. KOPA'H, ibid., I, p. 251) et "Chercheurs et législateurs de la langue ", Bibliothèque hellénique, $\mathrm{n}^{\circ}$ 6, Préface, 1812 (А. коРA'H, ibid., I, p. 309-310).

25. A. KORAïs, «Épictète », Bibliothèque hellénique, nº 7, Préface, 1827 (А. KOPA'H, ibid., I, p. 159).

26. Par exemple, I. Sycoutris ne pense pas que Koraïs puisse prétendre à un rôle plus profond que celui d'un simple philologue éditeur de textes anciens, alors que

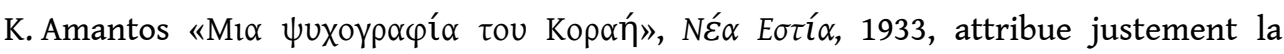
grandeur de Koraïs à l'importance que celui-ci accorda non seulement à la langue grecque, mais aussi à la valeur éthique de la culture hellénique (A. KOPA'H, ibid., I,

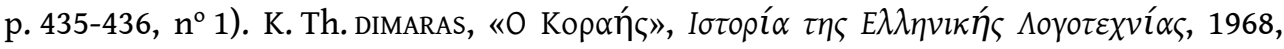
pour sa part, insiste sur le fondement politique des activités de Koraïs : la libération et l'éducation de la nation hellénique, qui passe par a connaissance des auteurs classiques de l'Antiquité (A. KOPA'H, ibid., I, p. 466-468).

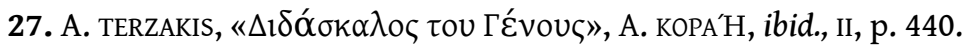




\section{RÉSUMÉS}

Adamantios Koraïs est un érudit cosmopolite de l'âge des Lumières qui, entre autres travaux, a traduit, commenté et publié de nombreux textes anciens. Koraïs pense que l'éducation est la clé du bien-être d'une personne et d'une nation. L'étude des grandes œuvres classiques de la littérature grecque lui semble appropriée pour réaliser cette tâche, difficile parmi toutes.

Dans le présent article, je tenterai de mieux clarifier ses positions sur la manière dont l'étude des auteurs « classiques » pouvait contribuer à l'éducation éthique et politique des Grecs, pendant la guerre d'indépendance et après la création de l'État néohellénique. Il est possible que les idées de Koraïs soient encore intéressantes aujourd'hui, pour tout être humain désirant une vie heureuse.

Adamantios Koraïs was a cosmopolitan scholar of the age of the Enlightenment, who translated, commented and published numerous ancient texts, among other works. Koraïs thinks that education is the key for the well being of a person and of a nation. The study of the great classical works of Greek literature seems appropriate to him for the realisation of this extremely difficult task.

In the present article I shall try to clarify better his positions on how could the study of the «classical » authors contribute to the ethical and political education of the Greeks, during the independence war and after the creation of the modern Greek state. It is possible that Koraïs ideas present still some interest today, for any human being who desires a happy life.

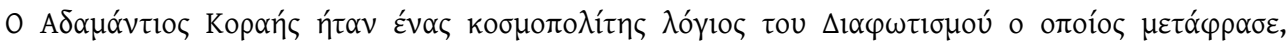

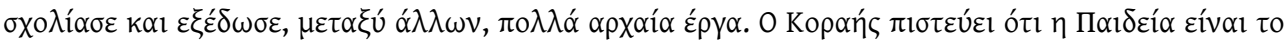

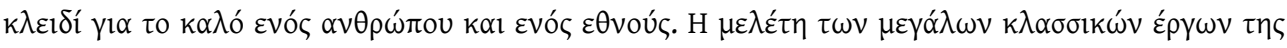

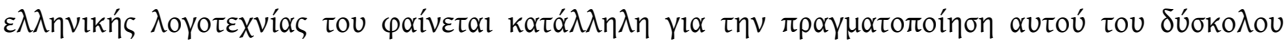
ह́pyou.

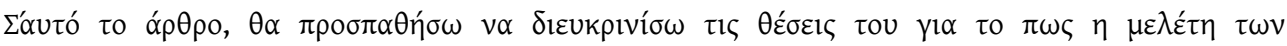

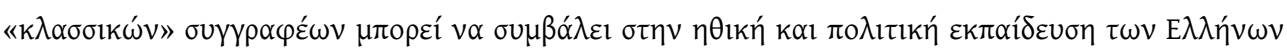



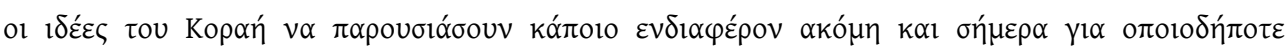

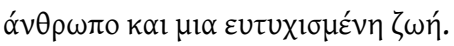

\section{INDEX}

Index géographique : Grèce

Mots-clés : Koraïs Adamantios (1748-1833), Koraïs Adamantios (1748-1833)

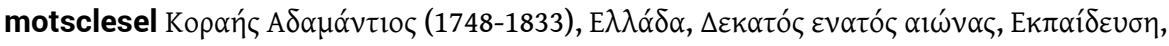

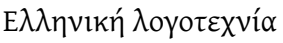

motsclestr Koraïs Adamantios (1748-1833), Yunanistan, Ondokuzuncu Yüzyıl, Eğitim, Yunan Edebiyat

motsclesmk КОРАИС АДАМАНТИОС (1748-1833), ГРЦИЈА, ДЕВЕТНАЕСЕТТИОТ ВЕК, ОБРАЗОВАНИЕ, ГРЧКАТА ЛИТЕРАТУРА

Thèmes : Éducation, Littérature grecque

Keywords : Koraïs Adamantios (1748-1833), Greece, Nineteenth century, Education, Greek literature

Index chronologique : dix-neuvième siècle 
AUTEUR

AIKATERINI LEFKA

Université de Liège 